\title{
Chronology of 1980s American Culture
}




\begin{tabular}{|c|c|c|c|c|}
\hline Date & Events & Criticism & Literature & Performance \\
\hline 1980 & $\begin{array}{l}\text { Jimmy Carter signs } \\
\text { Banking Deregulation } \\
\text { Act and Motor } \\
\text { Carrier Act } \\
\text { deregulating interstate } \\
\text { trucking. } \\
\text { Mount St Helens } \\
\text { erupts. } \\
\text { Military mission to } \\
\text { rescue fifty-two } \\
\text { hostages held at the } \\
\text { US Embassy in } \\
\text { Teheran fails. } \\
\text { US boycotts summer } \\
\text { Olympics in Moscow } \\
\text { in protest at Soviet } \\
\text { invasion of } \\
\text { Afghanistan. } \\
\text { Ronald Reagan } \\
\text { elected President. } \\
\text { Republicans control } \\
\text { the US Senate for the } \\
\text { first time since } 1956 . \\
\text { John Lennon shot } \\
\text { and killed by Mark } \\
\text { Chapman in New } \\
\text { York. } \\
\text { Rubik's Cube goes on } \\
\text { sale and Pac-Man } \\
\text { released. }\end{array}$ & $\begin{array}{l}\text { Milton and Rose } \\
\text { Friedman, Free to } \\
\text { Choose } \\
\text { Stephen Greenblatt, } \\
\text { Renaissance Self- } \\
\text { Fashioning } \\
\text { Victor Navasky, } \\
\text { Naming Names } \\
\text { Alvin Toffler, The } \\
\text { Third Wave } \\
\text { Immanuel } \\
\text { Wallerstein, The } \\
\text { Modern World- } \\
\text { System, Vol. II }\end{array}$ & $\begin{array}{l}\text { John Kennedy } \\
\text { Toole, A } \\
\text { Confederacy of } \\
\text { Dunces } \\
\text { E. L. Doctorow, In } \\
\text { Loon Lake } \\
\text { Marilynne } \\
\text { Robinson, } \\
\text { Housekeeping } \\
\text { Maxine Hong } \\
\text { Kingston, China } \\
\text { Men } \\
\text { James Schuyler, The } \\
\text { Morning of the } \\
\text { Poem }\end{array}$ & $\begin{array}{l}\text { Philip Glass, } \\
\text { Satyagraba } \\
\text { Kirke Mechem, } \\
\text { Tartuffe } \\
\text { Barnum } \\
\text { 42nd Street } \\
\text { Fame (Alan Parker) }\end{array}$ \\
\hline 1981 & $\begin{array}{l}\text { John Hinckley } \\
\text { attempts to } \\
\text { assassinate Ronald } \\
\text { Reagan. } \\
\text { The first space } \\
\text { shuttle, Columbia, is } \\
\text { launched. } \\
\text { US hostages released } \\
\text { in Tehran. } \\
\text { First cases of AIDS } \\
\text { identified. } \\
\text { The first American } \\
\text { test-tube baby is born } \\
\text { The IBM Personal } \\
\text { Computer goes on } \\
\text { sale. }\end{array}$ & $\begin{array}{l}\text { Stephen Jay Gould, } \\
\text { The Mismeasure of } \\
\text { Man } \\
\text { bell hooks, Ain't I a } \\
\text { Woman? } \\
\text { Fredric Jameson, } \\
\text { The Political } \\
\text { Unconscious } \\
\text { Tracy Kidder, The } \\
\text { Soul of a New } \\
\text { Machine } \\
\text { Tom Wolfe, From } \\
\text { Baubaus to Our } \\
\text { House }\end{array}$ & $\begin{array}{l}\text { Toni Morrison, Tar } \\
\text { Baby } \\
\text { John Updike, } \\
\text { Rabbit is Rich } \\
\text { David Bradley, The } \\
\text { Chaneysville } \\
\text { Incident } \\
\text { Leslie Marmon } \\
\text { Silko, Storyteller } \\
\text { Raymond Carver, } \\
\text { What We Talk } \\
\text { About When We } \\
\text { Talk About Love }\end{array}$ & $\begin{array}{l}\text { Split Britches lesbian } \\
\text { theatre company } \\
\text { formed } \\
\text { Dreamgirls }(1,522 \\
\text { performances) } \\
\text { Steve Reich, Tehillim } \\
\text { Tennessee Williams, } \\
\text { Something Cloudy, } \\
\text { Something Clear } \\
\text { The Jazz Singer } \\
\text { (Richard } \\
\text { Fleischer/Sidney J. } \\
\text { Furie) }\end{array}$ \\
\hline
\end{tabular}




\begin{tabular}{|c|c|c|}
\hline Film & Television & Music \\
\hline $\begin{array}{l}\text { Ordinary People } \\
\text { (Robert Redford) } \\
\text { The Empire Strikes } \\
\text { Back (George Lucas) } \\
\text { Raging Bull (Martin } \\
\text { Scorcese) } \\
\text { Coal Miner's Daughter } \\
\text { (Michael Apted) } \\
\text { Heaven's Gate } \\
\text { (Michael Cimino) }\end{array}$ & $\begin{array}{l}\text { Dallas (CBS, 1978-91) } \\
\text { The Dukes of Hazzard } \\
\text { (1979-85) } \\
\text { Knots Landing } \\
\text { (1979-1993) } \\
\text { Magnum, P.I. (CBS, } \\
\text { 1980-8) } \\
\text { Cable News Network } \\
\text { (CNN) begins } \\
\text { broadcasting }\end{array}$ & $\begin{array}{l}\text { Blondie, 'Call Me' } \\
\text { Lipps, Inc., } \\
\text { 'Funkytown' } \\
\text { Christopher Cross, } \\
\text { Christopher Cross } \\
\text { Kurtis Blow, Kurtis } \\
\text { Blow } \\
\text { Bruce Springsteen, The } \\
\text { River }\end{array}$ \\
\hline
\end{tabular}

Art

Various, 'Times Square Show'

Julian Schnabel, Exile

Fab 5 Freddy,

Campbell's Soup Cans, South Bronx, New York

Robert Mapplethorpe, Man in Polyester Suit Jasper Johns's Three Flags (1958) bought for $\$ 1$ million

Raiders of the Lost Ark (Steven Spielberg) Nine to Five (Colin Higgins) On Golden Pond (Mark Rydell) Wolfen (Michael Wadleigh)

Reds (Warren Beatty)
Hill Street Blues

(NBC, 1981-7)

Dynasty (ABC, 1981-9)

Falcon Crest (1981-90) The Fall Guy (1981-6) MTV is launched
Kim Carnes, 'Bette

Davis Eyes'

Blondie, 'Rapture'

Talking Heads, 'Once

in a Lifetime'

Mötley Crüe, Too Fast

For Love

Van Halen, Fair

Warning
Sherrie Levine, 'After Walker Evans' Mark Morrisoe, Untitled (Self-Portrait Standing in the Shower) Jean-Michel Basquiat, Untitled (Skull) Cindy Sherman, Centerfolds Richard Serra, Tilted Arc sculpture 


\begin{tabular}{|c|c|c|c|c|}
\hline Date & Events & Criticism & Literature & Performance \\
\hline 1982 & $\begin{array}{l}\text { De Lorean car factory } \\
\text { in Belfast goes into } \\
\text { receivership. } \\
\text { The first successful } \\
\text { artificial heart } \\
\text { transplant, at the } \\
\text { University of Utah } \\
\text { Medical Center. } \\
\text { Graceland, Elvis } \\
\text { Presley's Memphis } \\
\text { home, opened to the } \\
\text { public. } \\
\text { Vietnam Veterans } \\
\text { Memorial opens in } \\
\text { Washington. } \\
\text { Ronald Reagan } \\
\text { becomes first US } \\
\text { president to address } \\
\text { British parliament. } \\
\text { Time magazine's Man } \\
\text { of the Year is a } \\
\text { computer. }\end{array}$ & $\begin{array}{l}\text { Dennis Altman, The } \\
\text { Homosexualisation } \\
\text { of America } \\
\text { Jonathan Culler, On } \\
\text { Deconstruction } \\
\text { Jane Gallop, The } \\
\text { Daughter's } \\
\text { Seduction } \\
\text { Tom Peters and } \\
\text { Robert H. } \\
\text { Waterman Jr, In } \\
\text { Search of Excellence } \\
\text { Cornel West, } \\
\text { Prophesy } \\
\text { Deliverance! An } \\
\text { Afro-American } \\
\text { Revolutionary } \\
\text { Christianity }\end{array}$ & $\begin{array}{l}\text { Anne Tyler, Dinner } \\
\text { at the Homesick } \\
\text { Restaurant } \\
\text { Alice Walker, The } \\
\text { Color Purple } \\
\text { Don DeLillo, The } \\
\text { Names } \\
\text { Saul Bellow, The } \\
\text { Dean's December } \\
\text { Sylvia Plath, } \\
\text { Journals }\end{array}$ & $\begin{array}{l}\text { August Wilson, Ma } \\
\text { Rainey's Black } \\
\text { Bottom } \\
\text { Cats (7,485 } \\
\text { performances) } \\
\text { Charles Fuller, A } \\
\text { Soldier's Play } \\
\text { Little Shop of } \\
\text { Horrors (2,209 } \\
\text { performances) } \\
\text { Victor/Victoria } \\
\text { (Blake Edwards) }\end{array}$ \\
\hline 1983 & $\begin{array}{l}\text { Ronald Reagan } \\
\text { announces Star Wars } \\
\text { missile defence } \\
\text { initiative. } \\
241 \text { US Navy and } \\
\text { Marines personnel } \\
\text { killed in a suicide } \\
\text { bombing attack in } \\
\text { Beirut. } \\
\text { US invaded Grenada } \\
\text { to guard evacuations } \\
\text { of US citizens after a } \\
\text { Marxist takeover. } \\
\text { Sally Ride first } \\
\text { American woman in } \\
\text { space. } \\
\text { Final episode of } \\
M * A * S * H \text {. } \\
\text { US cruise missiles } \\
\text { arrive at Greenham } \\
\text { Common airbase in } \\
\text { Britain. }\end{array}$ & $\begin{array}{l}\text { Jonathan Arac, The } \\
\text { Yale Critics } \\
\text { Benedict Anderson, } \\
\text { Imagined } \\
\text { Communities } \\
\text { Manning Marable, } \\
\text { How Capitalism } \\
\text { Underdeveloped } \\
\text { Black America } \\
\text { Edward Said, The } \\
\text { World, the Text, and } \\
\text { the Critic } \\
\text { Susan Sheehan, Is } \\
\text { There No Place on } \\
\text { Earth for Me? }\end{array}$ & $\begin{array}{l}\text { Kathy Acker, Great } \\
\text { Expectations } \\
\text { William Kennedy, } \\
\text { Ironweed } \\
\text { Gary Snyder, } \\
\text { Passage Through } \\
\text { India } \\
\text { Ruth Prawer } \\
\text { Jhabvala, In Search } \\
\text { of Love and Beauty } \\
\text { Joan Didion, } \\
\text { Salvador }\end{array}$ & $\begin{array}{l}\text { Leonard Bernstein, } \\
\text { A Quiet Place } \\
\text { Baby (241 } \\
\text { performances) } \\
\text { Neil Simon, } \\
\text { Brighton Beach } \\
\text { Memoirs } \\
\text { Edward Albee, } \\
\text { Finding the Sun } \\
\text { Tina Howe, Painting } \\
\text { Churches }\end{array}$ \\
\hline
\end{tabular}




\begin{tabular}{|c|c|c|c|}
\hline Film & Television & Music & Art \\
\hline $\begin{array}{l}\text { E.T. the Extra- } \\
\text { Terrestrial (Steven } \\
\text { Spielberg) } \\
\text { On Golden Pond } \\
\text { (Mark Rydell) } \\
\text { Blade Runner (Ridley } \\
\text { Scott) } \\
\text { Tron (Steven Lisberger) } \\
\text { The Thing (John } \\
\text { Carpenter) }\end{array}$ & $\begin{array}{l}\text { St. Elsewhere (NBC, } \\
\text { 1982-8) } \\
\text { Knight Rider (NBC, } \\
\text { 1982-6) } \\
\text { Family Ties (NBC, } \\
1982-9) \\
\text { Cagney and Lacey } \\
\text { (CBS, 1982-8) } \\
\text { Cheers (NBC, 1982-3) }\end{array}$ & $\begin{array}{l}\text { Human League, 'Don't } \\
\text { You Want Me' } \\
\text { Survivor, 'Eye of the } \\
\text { Tiger' } \\
\text { Grandmaster Flash and } \\
\text { the Furious Five, 'The } \\
\text { Message' } \\
\text { Michael Jackson, } \\
\text { Thriller } \\
\text { Bruce Springsteen, } \\
\text { Nebraska }\end{array}$ & $\begin{array}{l}\text { Jenny Holzer, Truisms } \\
\text { (1982) } \\
\text { Jean-Michel Basquiat, } \\
\text { Two Heads on Gold } \\
\text { (1982) } \\
\text { David Salle exhibition } \\
\text { at Leo Castelli's } \\
\text { gallery, New York } \\
\text { Nan Goldin, Greer } \\
\text { and Robert on the Bed } \\
\text { Roni Horn, Gold Field }\end{array}$ \\
\hline
\end{tabular}

Trading Places (John Landis)

Flashdance (Adrian Lyne)

Star Wars Episode VI: Return of the Jedi (George Lucas) The King of Comedy (Martin Scorsese) Silkwood (Mike Nichols)
The A-Team (NBC, 1983-7)

$V$ (NBC mini-series) The Thorn Birds (ABC mini-series)

The Disney Channel is launched Country Music Television is launched
Irene Cara, 'Flashdance ... What a Feeling' The Police, 'Every Breath You Take' REM, Murmur Madonna, Madonna Metallica, Kill 'Em All
Richard Prince, Spiritual America Christo and JeanneClaude, Surrounded Islands, Biscayne Bay, Miami, Florida David Salle, Pitcher International Directory of Corporate Art Collections first published Shopping-mall developer Alfred Taubman takes over Sotheby's 


\begin{tabular}{|c|c|c|c|c|}
\hline Date & Events & Criticism & Literature & Performance \\
\hline 1984 & $\begin{array}{l}\text { The US re-established } \\
\text { full diplomatic } \\
\text { relations with the } \\
\text { Vatican for the first } \\
\text { time since } 1867 \text {. } \\
\text { Marvin Gaye shot } \\
\text { and killed by his } \\
\text { father. } \\
\text { Ronald Reagan re- } \\
\text { elected in biggest } \\
\text { Republican landslide } \\
\text { in US voting history. } \\
\text { Agent Orange Fund } \\
\text { for Vietnam veterans } \\
\text { started by chemical } \\
\text { companies. } \\
\text { Soviet Union } \\
\text { boycotts Olympic } \\
\text { Games in Los } \\
\text { Angeles. } \\
\text { Velma Barfield } \\
\text { becomes first woman } \\
\text { to be executed in the } \\
\text { US since } 1962\end{array}$ & $\begin{array}{l}\text { Christopher Lasch, } \\
\text { The Minimal Self } \\
\text { Mark McCormack, } \\
\text { What They Don't } \\
\text { Teach You at } \\
\text { Harvard Business } \\
\text { School } \\
\text { Fredric Jameson, } \\
\text { 'Postmodernism, or, } \\
\text { The Cultural Logic } \\
\text { of Late Capitalism' } \\
\text { Charles Murray, } \\
\text { Losing Ground } \\
\text { Studs Terkel, The } \\
\text { Good War }\end{array}$ & $\begin{array}{l}\text { William Gibson, } \\
\text { Neuromancer } \\
\text { Sonia Sanchez, } \\
\text { Homegirls and } \\
\text { Handgrenades } \\
\text { Gore Vidal, Lincoln } \\
\text { Thomas Pynchon, } \\
\text { Slow Learner } \\
\text { (collection of early } \\
\text { short stories) } \\
\text { Robert Creeley, The } \\
\text { Collected Prose of } \\
\text { Robert Creeley }\end{array}$ & $\begin{array}{l}\text { David Mamet, } \\
\text { Glengarry Glen Ross } \\
\text { Sunday in the Park } \\
\text { with George (604 } \\
\text { performances) } \\
\text { Philip Glass, } \\
\text { Akhnaten } \\
\text { August Wilson, Joe } \\
\text { Turner's Come and } \\
\text { Gone } \\
\text { The Cotton Club } \\
\text { (Francis Ford } \\
\text { Coppola) }\end{array}$ \\
\hline 1985 & $\begin{array}{l}\text { Mikhail Gorbachev } \\
\text { becomes leader of } \\
\text { Soviet Union. Reagan } \\
\text { and Gorbachev meet } \\
\text { later in the year. } \\
\text { Rock Hudson dies } \\
\text { from AIDS-related } \\
\text { illnesses. } \\
\text { First commercial } \\
\text { (.com) domain names } \\
\text { registered on the } \\
\text { Internet Domain } \\
\text { Name System. } \\
\text { Live Aid concerts in } \\
\text { London and } \\
\text { Philadelphia. } \\
\text { Coca-Cola } \\
\text { unsuccessfully tries to } \\
\text { change its recipe. } \\
\text { Microsoft releases } \\
\text { first version of } \\
\text { Windows. }\end{array}$ & $\begin{array}{l}\text { T. J. Jackson Lears, } \\
\text { 'The Concept of } \\
\text { Cultural Hegemony' } \\
\text { J. Anthony Lukas, } \\
\text { Common Ground } \\
\text { Richard Slotkin, The } \\
\text { Fatal Environment } \\
\text { Eve Kosofsky } \\
\text { Sedgwick, Between } \\
\text { Men } \\
\text { Walter Benn } \\
\text { Michaels and } \\
\text { Donald Pease, The } \\
\text { American } \\
\text { Renaissance } \\
\text { Reconsidered }\end{array}$ & $\begin{array}{l}\text { Paul Auster, City of } \\
\text { Glass } \\
\text { Don DeLillo, White } \\
\text { Noise } \\
\text { Bharati Mukherjee, } \\
\text { Darkness } \\
\text { Cormac McCarthy, } \\
\text { Blood Meridian } \\
\text { Bret Easton Ellis, } \\
\text { Less Than Zero }\end{array}$ & $\begin{array}{l}\text { Sam Shepard, A Lie } \\
\text { of the Mind } \\
\text { August Wilson, } \\
\text { Fences } \\
\text { Larry Kramer, The } \\
\text { Normal Heart } \\
\text { Dorothy Rudd } \\
\text { Moore, Frederick } \\
\text { Douglass } \\
\text { Terence McNally, } \\
\text { The Lisbon Traviata }\end{array}$ \\
\hline
\end{tabular}




\begin{tabular}{|c|c|c|c|}
\hline Film & Television & Music & Art \\
\hline $\begin{array}{l}\text { Ghostbusters (Ivan } \\
\text { Reitman) } \\
\text { Indiana Jones and the } \\
\text { Temple of Doom } \\
\text { (Steven Spielberg) } \\
\text { The Terminator (James } \\
\text { Cameron) } \\
\text { Paris, Texas (Wim } \\
\text { Wenders) } \\
\text { A Nightmare on Elm } \\
\text { Street (Wes Craven) }\end{array}$ & $\begin{array}{l}\text { Miami Vice (NBC, } \\
\text { 1984-9) } \\
\text { The Cosby Show } \\
\text { (NBC, 1984-92) } \\
\text { Murder, She Wrote } \\
\text { (CBS, 1984-96) } \\
\text { Kate and Allie (CBS, } \\
\text { 1984-9) } \\
\text { Santa Barbara (NBC, } \\
1984-93 \text { ) }\end{array}$ & $\begin{array}{l}\text { Van Halen, 'Jump' } \\
\text { Prince, 'When Doves } \\
\text { Cry' } \\
\text { Hüsker Dü, Zen } \\
\text { Arcade } \\
\text { Madonna, Like a } \\
\text { Virgin } \\
\text { Bruce Springsteen, } \\
\text { Born in the U.S.A. }\end{array}$ & $\begin{array}{l}\text { Louise Lawler, Pollock } \\
\text { and Tureen, Arranged } \\
\text { by Mr. and Mrs. } \\
\text { Burton Tremaine, } \\
\text { Connecticut (1984) } \\
\text { Robert Longo, Tongue } \\
\text { to the Heart } \\
\text { Jean-Michel Basquiat, } \\
\text { Flexible } \\
\text { The Border Art } \\
\text { Workshop established } \\
\text { Chase Manhattan Bank } \\
\text { acquired } 1,300 \text { pieces } \\
\text { of art for its collection }\end{array}$ \\
\hline
\end{tabular}

Back To The Future

(Robert Zemeckis)

Out Of Africa (Sydney

Pollack)

The Breakfast Club

(John Hughes)

Desperately Seeking

Susan (Susan

Seidelman)

Rambo: First Blood

Part II (George P.

Cosmatos)
The Golden Girls

(NBC, 1985-92)

Moonlighting (ABC,

1985-9)

North and South

(ABC, mini-series)

The Equalizer (1985-9)

Discovery Channel is

launched
USA for Africa, 'We

Are the World'

Dire Straits, 'Money

for Nothing'

Megadeth, Killing Is

My Business ... And

Business Is Good!

Phil Collins, No Jacket

Required

LL Cool J, Radio
Jeff Koons, One Ball Total Equilibrium

Tank

Keith Haring, 'The Ten

Commandments'

Robert Mapplethorpe,

Lindsay Key

PC Paintbrush

software first released

Fractal art emerges

after publication of

Scientific American

article on Mandelbrot

Set 


\begin{tabular}{|c|c|c|c|c|}
\hline Date & Events & Criticism & Literature & Performance \\
\hline 1986 & $\begin{array}{l}\text { Space shuttle } \\
\text { Challenger explodes } \\
\text { shortly after take-off. } \\
\text { Iran-Contra scandal. } \\
\text { Clint Eastwood } \\
\text { elected Republican } \\
\text { mayor of Carmel, } \\
\text { California. } \\
\text { First personal- } \\
\text { computer virus } \\
\text { discovered. } \\
\text { Chernobyl nuclear } \\
\text { disaster. } \\
\text { Failure of nuclear } \\
\text { arms reduction talks } \\
\text { in Reykjavik, Iceland, } \\
\text { between US and } \\
\text { Soviet Union. } \\
\text { Mike Tyson become } \\
\text { world heavyweight } \\
\text { boxing champion. }\end{array}$ & $\begin{array}{l}\text { Cathy Davidson, } \\
\text { Revolution and the } \\
\text { Word } \\
\text { Barry Lopez, Arctic } \\
\text { Dreams } \\
\text { Janice Radway, } \\
\text { 'Identifying } \\
\text { Ideological Seams' } \\
\text { Russell Reising, The } \\
\text { Unusable Past. } \\
\text { Richard Rhodes, } \\
\text { Making of the } \\
\text { Atomic Bomb }\end{array}$ & $\begin{array}{l}\text { Ann Beattie, Where } \\
\text { You'll Find Me } \\
\text { Art Spiegelman, } \\
\text { Maus } \\
\text { Philip Roth, The } \\
\text { Counterlife } \\
\text { Richard Ford, The } \\
\text { Sportswriter } \\
\text { Kathy Acker, Don } \\
\text { Quixote }\end{array}$ & $\begin{array}{l}\text { Me and My Girl } \\
\text { (1,420 performances) } \\
\text { August Wilson, The } \\
\text { Piano Lesson } \\
\text { Sweet Charity ( } 369 \\
\text { performances) } \\
\text { George Furth, } \\
\text { Precious Sons } \\
\text { A. R. Gurney, The } \\
\text { Perfect Party }\end{array}$ \\
\hline 1987 & $\begin{array}{l}\text { Ronald Reagan } \\
\text { undergoes prostate } \\
\text { surgery. } \\
\text { Tower Commission } \\
\text { Report published on } \\
\text { Iran-Contra scandal. } \\
\text { Single European Act } \\
\text { passed by the } \\
\text { European Union. } \\
\text { Black Monday stock } \\
\text { market crash. } \\
\text { Death of Andy } \\
\text { Warhol. } \\
\text { Prozac released in the } \\
\text { US. }\end{array}$ & $\begin{array}{l}\text { Allan Bloom, The } \\
\text { Closing of the } \\
\text { American Mind } \\
\text { Michael Denning, } \\
\text { Mechanic Accents } \\
\text { James Gleick, Chaos } \\
\text { Randy Shilts, And } \\
\text { the Band Played On } \\
\text { Gayatri } \\
\text { Chakravorty Spivak, } \\
\text { In Other Worlds }\end{array}$ & $\begin{array}{l}\text { Stephen King, } \\
\text { Misery } \\
\text { Toni Morrison, } \\
\text { Beloved } \\
\text { Tom Wolfe, Bonfire } \\
\text { of the Vanities } \\
\text { Scott Turow, } \\
\text { Presumed Innocent } \\
\text { James Ellroy, Black } \\
\text { Dablia }\end{array}$ & $\begin{array}{l}\text { Les Misérables } \\
\text { (6,680 performances) } \\
\text { Starlight Express } \\
\text { ( } 761 \text { performances) } \\
\text { Into the Woods ( } 765 \\
\text { performances) } \\
\text { John Adams, Nixon } \\
\text { in China } \\
\text { Cabaret (revival, } 261 \\
\text { performances) }\end{array}$ \\
\hline
\end{tabular}




\begin{tabular}{|c|c|c|c|}
\hline Film & Television & Music & Art \\
\hline $\begin{array}{l}\text { Top Gun (Tony Scott) } \\
\text { Ferris Bueller's Day } \\
\text { Off (John Hughes) } \\
\text { Aliens (James } \\
\text { Cameron) } \\
\text { Blue Velvet (David } \\
\text { Lynch) } \\
\text { Manbunter (Michael } \\
\text { Mann) }\end{array}$ & $\begin{array}{l}\text { The Oprah Winfrey } \\
\text { Show (syndicated, } \\
\text { 1986-present) } \\
\text { L.A. Law (NBC, } \\
\text { 1986-94) } \\
\text { Space Shuttle } \\
\text { Challenger explodes on } \\
\text { live television } \\
\text { Fox Broadcasting } \\
\text { Company is launched } \\
\text { Boy George appears in } \\
\text { The A-Team }\end{array}$ & $\begin{array}{l}\text { Whitney Houston, } \\
\text { 'Greatest Love of All' } \\
\text { Prince and the } \\
\text { Revolution, 'Kiss' } \\
\text { Run-DMC, Raising } \\
\text { Hell } \\
\text { Slayer, Reign in Blood } \\
\text { Janet Jackson, Control }\end{array}$ & $\begin{array}{l}\text { Jeff Koons, Rabbit } \\
\text { Gran Fury, Silence = } \\
\text { Death } \\
\text { Judy Pfaff, Wasco } \\
\text { Julian Schnabel, Self- } \\
\text { Portrait in Andy's } \\
\text { Shadow } \\
\text { Mark Tansey, Forward } \\
\text { Retreat }\end{array}$ \\
\hline
\end{tabular}

Fatal Attraction

(Adrian Lyne)

Wall Street (Oliver

Stone)

Dirty Dancing (Emile

Ardolino)

The Fly (David

Cronenberg)

Full Metal Jacket

(Stanley Kubrick) thirtysomething (ABC, Bon Jovi, 'Livin'on a 1987-91)

Teenage Mutant Ninja Turtles (syndicated, 1987-96)

Star Trek: The Next

Generation

(syndicated, 1987-94)

Amerika (ABC, miniseries)

The Simpsons first appears as a series of short animations on The Tracey Ullman Show
Prayer'

Bill Medley and Jennifer Warnes, '(I’ve $\mathrm{Had}$ ) The Time of My Life' Public Enemy, Yo! Bum Rush the Show Joe Satriani, Surfing with the Alien Jane's Addiction, Jane's Addiction
David Wojnarowicz, Seven Miles a Second Andreas Serrano, Piss Christ Julian Schnabel, Grace High Tower

Andy Warhol dies while undergoing gallbladder surgery Roman Verostko pioneers algorithmic art using a plotter connected to a paintbrush 


\begin{tabular}{|c|c|c|c|c|}
\hline Date & Events & Criticism & Literature & Performance \\
\hline 1988 & $\begin{array}{l}\text { Soviet Union begins } \\
\text { withdrawal from } \\
\text { Afghanistan. } \\
\text { House of } \\
\text { Representatives } \\
\text { refuses Ronald } \\
\text { Reagan request for } \\
\text { financial support of } \\
\text { Contras in Nicaragua. } \\
\text { Iran-Iraq war ends } \\
\text { after eight years. } \\
\text { Space shuttle flights } \\
\text { resume after } \\
\text { Challenger disaster. } \\
\text { George H. W. Bush } \\
\text { defeats Michael } \\
\text { Dukakis to become } \\
\text { US president. } \\
\text { Pan Am flight } 103 \\
\text { from London to New } \\
\text { York explodes over } \\
\text { Scottish town of } \\
\text { Lockerbie. }\end{array}$ & $\begin{array}{l}\text { Jean Baudrillard, } \\
\text { America } \\
\text { Sacvan Bercovitch } \\
\text { and Myra Jehlen, } \\
\text { Ideology and Classic } \\
\text { American Literature } \\
\text { Stanley Cavell, In } \\
\text { Quest of the } \\
\text { Ordinary } \\
\text { Catherine } \\
\text { McKinnon and } \\
\text { Andrea Dworkin, } \\
\text { Pornography and } \\
\text { Civil Rights } \\
\text { Neil Sheehan, A } \\
\text { Bright Shining Lie }\end{array}$ & $\begin{array}{l}\text { Kathy Acker, } \\
\text { Empire of the } \\
\text { Senseless } \\
\text { Nicholson Baker, } \\
\text { The Mezzanine } \\
\text { Don DeLillo, Libra } \\
\text { Raymond Carver, } \\
\text { Where I'm Calling } \\
\text { From } \\
\text { Annie Proulx, } \\
\text { Heartsongs and } \\
\text { Other Stories }\end{array}$ & $\begin{array}{l}\text { The Phantom of the } \\
\text { Opera }(7,709 \\
\text { performances at } 23 \\
\text { July 2006) } \\
\text { Wendy Wasserstein, } \\
\text { The Heidi } \\
\text { Chronicles } \\
\text { David Mamet, } \\
\text { Speed-the-Plow } \\
\text { Madame Butterfly } \\
\text { (777 performances) } \\
\text { Sarafina (597 } \\
\text { performances) }\end{array}$ \\
\hline 1989 & $\begin{array}{l}\text { Serial killer Ted } \\
\text { Bundy executed in } \\
\text { Florida. } \\
\text { Exxon Valdez oil } \\
\text { tanker runs aground } \\
\text { in Alaska spilling } \\
240,000 \text { gallons of oil. } \\
\text { Tiananmen Square } \\
\text { protests in Beijing, } \\
\text { China. } \\
\text { Revolutions across } \\
\text { Central and Eastern } \\
\text { Europe see } \\
\text { communist } \\
\text { governments } \\
\text { overthrown. } \\
\text { Dilbert comic strip } \\
\text { published for the first } \\
\text { time. } \\
\text { First sales of } \\
\text { Nintendo Game Boy. }\end{array}$ & $\begin{array}{l}\text { Richard Rorty, } \\
\text { Contingency, Irony, } \\
\text { Solidarity } \\
\text { Henry Louis Gates } \\
\text { Jr, The Signifying } \\
\text { Monkey } \\
\text { Donna Haraway, } \\
\text { Primate Visions } \\
\text { Francis Fukuyama, } \\
\text { 'The End of } \\
\text { History?' } \\
\text { Immanuel } \\
\text { Wallerstein, The } \\
\text { Modern World- } \\
\text { System, Vol. III }\end{array}$ & $\begin{array}{l}\text { Maxine Hong } \\
\text { Kingston, } \\
\text { Tripmaster Monkey } \\
\text { Bharati Mukherjee, } \\
\text { Jasmine } \\
\text { Amy Tan, The Joy } \\
\text { Luck Club } \\
\text { New Revised } \\
\text { Standard Version of } \\
\text { The Bible } \\
\text { Paul Auster, Moon } \\
\text { Palace }\end{array}$ & $\begin{array}{l}\text { City of Angels ( } 878 \\
\text { performances) } \\
\text { Conrad Cummings, } \\
\text { Photo-Op } \\
\text { Anthony Davis, } \\
\text { Under the Double } \\
\text { Moon } \\
\text { Mandy Patinkin in } \\
\text { Concert: 'Dress } \\
\text { Casual' } \\
\text { Meet Me in St. Louis } \\
\text { (revival, 252 } \\
\text { performances) }\end{array}$ \\
\hline
\end{tabular}




\begin{tabular}{|c|c|c|c|}
\hline Film & Television & Music & Art \\
\hline $\begin{array}{l}\text { Rain Man (Barry } \\
\text { Levinson) } \\
\text { Who Framed Roger } \\
\text { Rabbit (Robert } \\
\text { Zemeckis/Richard } \\
\text { Williams) } \\
\text { Die Hard (John } \\
\text { McTiernan) } \\
\text { Working Girl (Mike } \\
\text { Nichols) } \\
\text { The Accused (Jonathan } \\
\text { Kaplan) }\end{array}$ & $\begin{array}{l}\text { Roseanne (ABC, } \\
\text { 1988-97) } \\
\text { The Wonder Years } \\
\text { (ABC, 1988-93) } \\
\text { Murphy Brown (CBS, } \\
\text { 1988-98) } \\
\text { America's Most Wanted } \\
\text { (Fox, 1988-present) } \\
\text { Turner Network } \\
\text { Television is launched }\end{array}$ & $\begin{array}{l}\text { Guns N' Roses, 'Sweet } \\
\text { Child O' Mine' } \\
\text { Rick Astley, 'Never } \\
\text { Gonna Give You Up' } \\
\text { Public Enemy, It Takes } \\
\text { a Nation of Millions to } \\
\text { Hold Us Back } \\
\text { R.E.M., Green } \\
\text { Tracy Chapman, Tracy } \\
\text { Chapman }\end{array}$ & $\begin{array}{l}\text { Julian Schnabel, Joe } \\
\text { Glassco } \\
\text { Jeff Koons, Michael } \\
\text { Jackson and Bubbles } \\
\text { John Baldessari, } \\
\text { Seashells/Tridents/Fra } \\
\text { mes } \\
\text { Roman Verostko, } \\
\text { Pathway } 1 \\
\text { Jean-Michel Basquiat } \\
\text { dies of a heroin } \\
\text { overdose }\end{array}$ \\
\hline
\end{tabular}

\begin{tabular}{|c|c|c|c|}
\hline $\begin{array}{l}\text { Batman (Tim Burton) } \\
\text { Do the Right Thing } \\
\text { (Spike Lee) } \\
\text { Honey, I Shrunk the } \\
\text { Kids (Joe Johnston) } \\
\text { Glory (Edward Zwick) } \\
\text { Heathers (Michael } \\
\text { Lehmann) }\end{array}$ & $\begin{array}{l}\text { Seinfeld (NBC, } \\
\text { 1989-98) } \\
\text { The Simpsons (Fox, } \\
\text { 1989-present) } \\
\text { Baywatch (NBC, } \\
\text { 1989-2001) } \\
\text { America's Funniest } \\
\text { Home Videos (ABC, } \\
\text { 1989-present) } \\
\text { 1956 Christmas special } \\
\text { episode of I Love Lucy }\end{array}$ & $\begin{array}{l}\text { Madonna, 'Like a } \\
\text { Prayer' } \\
\text { The Bangles, 'Eternal } \\
\text { Flame' } \\
\text { De La Soul, } 3 \text { Feet } \\
\text { High and Rising } \\
\text { Fugazi, } 13 \text { Songs } \\
\text { N.W.A., Straight } \\
\text { Outta Compton }\end{array}$ & $\begin{array}{l}\text { Richard Prince, } \\
\text { Untitled (Cowboy) } \\
\text { Group Material, AIDS } \\
\text { Timeline (1989-91) } \\
\text { Gran Fury, Kissing } \\
\text { Doesn't Kill: Greed } \\
\text { and Indifference Do } \\
\text { Robert Mapplethorpe, } \\
\text { 'The Perfect Moment' } \\
\text { Cindy Sherman, } \\
\text { History Portraits }\end{array}$ \\
\hline
\end{tabular}


